\title{
ENGINEERING
}

\section{On The Employment Of The Personal Plant's Protect Connexion To Take Care Of The After Harvest Fruit Variety}

\author{
Prof. A.G. Sanchez, Prof. J.M. Jimenez Orea \\ Department Of Agriculture Science University Of Granada, Spain
}

\begin{abstract}
This work demonstrates that the personalplant protect compounds, like resveratrol in grapes, may be used as a natural and ecological various to chemical pesticides. associate degree extract from tracheophyte leaves was accustomed maintain the standard of post-harvested grapes. Specifically, associate degree extract ( $95 \%$ water and five-hitter ethanol) of such leaves was ready and, afterwards, completely different bunches of grapes were immersed in it. an equivalent range of bunches received either water or plant product (5\%) treatment, being each used as blank. Remarkably, on the fourteenth day when the start of the treatment the extract treated grapes, forever maintained at temperature, showed no sign of physical deterioration. In distinction, the 2 blank samples, i.e.: the water or plant product treated bunches appeared dehydrated, infected and deteriorated. moreover, the external application of the leaf's extract doesn't modify sensory, organic chemistry and nutritionary properties of the fruits. The useful effects of trans-resveratrol, like antitumor, antiviral, neuroprotective, antiaging and antiinflamatory support additional the employment of this natural compound rather than dangerous artificial pesticides.
\end{abstract}

Keywords: Weighted Mixtures, Phytoalexines, After Harvest, Fruit Treatment, Fruit Variety. 


\section{ENGINEERING}

\section{Introduction}

In most climates, fruits will solely be fully grpersonaland harvested throughout alittle a part of the year. Yet, consumption of fruit, and thus demand happens throughout the year. For this reason, giant elements of fruit harvests should be keep for extended periods of your time before they're sold to the customers. Obviously, such storage causes extended losses because of infectious agent attack and natural senescence. the normal solutions to those issues are storage underneath controlled or changed atmospheres and therefore the use of artificial pesticides. however, losses extraordinary two hundredth aren't uncommon. With relevance the employment of controlled or changed atmospheres, tho' vital advances are revamped the recent years $[1,2]$, no general and economical methodology is nevertheless on the market.

In this work we tend to explore another to the employment of each controlled atmospheres and chemical pesticides. Our approach consists of mistreatment the plant personalpesticides to boost their natural resistance. Natural merchandise of plant's secondary metabolism are employed in "natural" drugs since the first times of human history. a crucial cluster of secondary metabolites square measure the alleged "phytoalexines": antiphatogenic connexionmade by plants when infection or stimulation by organic phenomenon or abiotic agents. underneath infectious agent attack plants evolve refined systems of detection and response to decipher the infectious agent signals and induce acceptable defenses. These systems embrace specific networks that operate through the action of sign molecules like NSAID, jasmonate and olefin and generate the buildup of infectious agent connected proteins, phytoalexins or different phenoplast connexion

\section{Materials And Ways}

\section{FTIR Analysis}

Because of skillfulness and ease, we've utilized the Fourier remodel Infrared chemical analysis (FTIR) to analyse and characterize the tracheophyte leaves. however, a additional refined optical maser analytical technique [30] (see below for additional details) was accustomed ensure the presence of the compound, tho' is accessibility isn't necessary for the protocol here developed, as explained belowTo prepare the sample for FTIR analysis, tracheophyte leaves were dried 


\section{THE AMERICAN JOURNAL OF}

AGRICULTURE AND BIOMEDICAL

VOLUME01 ISSUE04

\section{ENGINEERING}

before grinding them to particle size below twenty five $\mathrm{m}$. afterward a combination with $\mathrm{KBr}$ was ready in a very weight share of four-dimensional and ninety six for sample and $\mathrm{KBr}$ elements, severally. Once the ready mixture was uniform one hundred $\mathrm{mg}$ of it absolutely was used for every run of transmission or diffuse reflectivity measurements.

\section{Extracts Preparation and Grapes Treatment}

The experiments here conferred were meted out with associate degree ethanolic extract obtained from Aledo tracheophyte leaves. This selection was designated as a result of its high concentration of transresveratrol (typically ten ppm). so as to get such extract, the leaves were cut in little items and stuffed in a very recipient with plant product. The quantities used were eight $L$ of plant product to extract three. 5 metric weight unit of tracheophyte leaves. The leaves were maintained in plant product with no sample agitation and light-weight protected throughout seven weeks to make sure the whole extraction of the phenoplast compounds. sporadically, a sample was taken and analized by UV-visible and FTIR spectrophotometry to watch the advance of the polyphenolic connexionextraction.

\section{Laser action and Resonant lonization Mass spectroscopy}

The trans-resveratrol content within the tracheophyte leaves was measured mistreatment the optical maser technique developed in our research lab that has been wide represented elsewhere $[25,30]$ thus solely a short report is given here. it's supported the mix of optical maser action (LD) with optical maser Resonance-Enhanced Multi-Photon Ionisation (REMPI) coupled to Time-of-Flight Mass spectroscopy (TOFMS) detection. A basic feature of the technique is that the absence of any separation technique for sample preparation, thus, the mix of optical maser action followed by REMPI-TOFMS detection will overcome the most error sources, gift within the chromatographical ways typically utilized for trans-resveratrol analysis in advanced samples.

\section{Microbiological Analysis}




\section{ENGINEERING}

Weighted mixtures of grapes skin (50\%) and tracheophyte leaves (50\%) were ground and diluted in a very sterile isotonic solution of $\mathrm{NaCl}$ 11th of September. Grape skins and leaves were employed in the microbiological work for completeness. However, similar results were obtained by using grape skins or leaves alone. The polyphenols' content (pc) of every mixture was monitored by the intensity of the $3300-3000 \mathrm{~cm}-1$ band within the various FTIR spectrum. 2 mixtures knpersonalas $A$ and $B$ were designated specified their pcA / pcB quantitative relation was ca. 4. moreover, samples were ready determination three g of every mixture in thirty metric capacity unit of the $\mathrm{NaCl}$ answer, then ordered decimal dilu.

\section{Results And Discussion}

The Fourier remodel spectrum of various samples: particularly, pure stilbene (top panel), pure trans-resveratrol (bottom panel) and tracheophyte leaf powder. an equivalent share of sample (4\%) and $\mathrm{KBr}(96 \%)$ was used for every analysis. because of the presence of the resveratrol within the dried tracheophyte leaf, its spectral band shows additional similarity thereto of pure resveratrol than thereto of pure stilbene. To additional ensure the presence of the resveratrol within the dried tracheophyte leaf, we've applied our optical maser analytical technique to the samples; the spectrum obtained with this technique; the peaks similar to stilbene (added as internal reference), resveratrol and quercitrin may be noticed.

Sensory and organic chemistry analysis of those samples were enforced following a typical procedure already represented elsewhere [28]. The results indicate that the external application of this extract, from the personalplant's leaf, doesn't modify sensory, organic chemistry and nutritionary properties of the treated fruits compared with those of non-treated, ones.

To demonstrate the improved natural resistance of the post-harvest grapes is because of the antifungic and antimicrobiological character of polyphenols content gift within the tracheophyte leaves a microbiological take a look at was do. Following the microbiological analysis protocol represented in Material and Section ways and mistreatment an equivalent amount of each $A$ and $B$ samples, the various colony forming units per gram were counted when sample incubation in potato-dextroseagar. 


\section{THE AMERICAN JOURNAL OF}

AGRICULTURE AND BIOMEDICAL

\section{VOLUME01 ISSUE04}

\section{ENGINEERING}

\section{Summary And Conclusions}

The most conclusion of the current work is that the vital improvement of the post-harvest resistance of grapes once these fruits square measure immersed in a very answer fashioned by a ninety fifth water and five-hitter plant product tracheophyte leaves' extract. Thus, it absolutely was outstanding to look at that whereas at the fourteenth day of treatment the extract - treated grapes maintained a physical facet with no sign of decay, the 2 different samples, i.e.: the wateR.

The ascertained positive influence of the natural extracts has been attributed to the presence of polyphenols as monitored by their IR optical phenomenon and incontestible by specific microbiological analysis. Indeed, analysis performed with these natural samples clearly incontestible however the upper the content of polyphenols the lower the quantity of colony forming units when correct cultivation in adequate growing media.

\section{References}

1. Nrtés-Hernández F, Artés F, Tomás-Barberán solfa syllable. Varietyy and sweetening of bioactive phenolics in CV. Napoleon table grapes exposed to completely different after harvestgassy treatments. J Agric Food Chem 2000.

2. Vaurus Ballesta $M T$, Jiménez JB, Romero I, et al. impact of high dioxide pretreatment on varietyy, plant decay and molecular regulation of stilbene phytoalexin biogenesis in keep table grapes. After harvestBiol Technol 2001.

3. Kutchan metal. Ecological Arsenal and organic process dispatcher. the paradigm of secondary metabolism. Plant Physiol 1998.

4. J. Mhytoalexins, Stress metabolism, and malady resistance in plants. Ann Rev Phytopathol 1990. 


\section{THE AMERICAN JOURNAL OF}

\section{AGRICULTURE AND BIOMEDICAL}

\section{VOLUME01 ISSUE04}

\section{ENGINEERING}

5. H. Phytoalexins: wath have we tend to learned when sixty nine years? Annu Rev Phytopathol 1995.

6. Miller DM, Douglas TJ. Development of ways for screening gravepines for resistance to false mildew. II.- Resveratrol production. Am J organic compound Vitic 1997.

7. LL, Coffee M. Phytoalexin production potential of grape berries. J Am Soc Hortic Sci 1986.

8. Cryce RJ. the assembly of resveratrol and therefore the viniferins by gravepines in response to ultraviolet irradiation. Phytochemistry 1987. 